\title{
Review on swine flu and status of swine flu in ethiopia
}

\begin{abstract}
Swine flu is a highly contagious respiratory viral disease which is caused by influenza a virus. The disease can be found everywhere in the world especially in those peoples rearing pigs. The virus can circulate between human, avian, and swine. It spread through aerosol transmission, via contact with affected pigs and contaminated objects with the virus. Different factors including immuno suppression predispose individuals to the disease. Swine influenza manifested by coughing, anorexia and lethargy in pigs while sudden fever and cough in human. The disease can be diagnosed either by laboratory or by observing signs and symptoms. It has a devastating effect on the economy and health of the communities even though it can be prevented and controlled with different measures like vaccines and antiviral drugs. Recently, active case reported in Addis Ababa, Ethiopia. So, due to its highly spread and economic importance it should have to be reported immediately to authorities if the cases have been seen and care should be taken if person showing the sign of the disease is observed.
\end{abstract}

Keywords: Ethiopia, influenza a virus, pandemic, swine, swine flu, laboratory, coughing, anorexia, lethargy, fever, diagnosed
Volume 4 Issue 2 - 2017

Sagni Ragasa, Eyob Hirpa

Wollega University School of Veterinary Medicine, Ethiopia

Correspondence: Eyob Hirpa, Wollega University School of Veterinary Medicine, PO Box 395, Wollega, Ethiopia, Nekemte, Ethiopia, Tel +25 I933686525, Email eyobresearch@gmail.com

Received: January II, 2017| Published: March 14, 2017
Abbreviations: SIV, swine influenza virus; SOIV, swine origin influenza virus; ARDS, acute respiratory disease syndrome; RT-PCR, reverse transcriptase-polymerase chain reaction; HA, hemagglutinin; NA, nuraminidase; ILI, influenza like illness; USFDA, united states food and drug administration; $\mathrm{MOH}$, ministry of health; SARI, severe acute respiratory infections

\section{Introduction}

Swine is a very important animal for the socio-economic improvement of the community as of any other livestock. However, the health disorder in these animals brings the major problem that greatly affects their production and productivity. Pigs can be infected by different types of diseases of various origins including viruses. Influenza, which is known as "the flu", is an infectious disease caused by the influenza virus, ${ }^{1-3}$ is one of the most prevalent respiratory viruses responsible for annual epidemics as well as occasional pandemics have a devastating results ${ }^{4,5}$ Swine flu, also called pig influenza, swine influenza, hog flu and pig flu which is caused by swine influenza virus (SIV) or swine-origin influenza virus (SOIV) have a great economic and public heath significance having genomes those affect swine, avian and human. ${ }^{6}$ Swine have the unique characteristic of being host to both human as well as avian species thus serving as 'mixing hosts' in which new strains adapted to humans. ${ }^{7}$ Due to the nature of respiratory virus, the transmission of this pathogenic virus is via air and hence, there is rapid spreading and difficulty in control of this infection. Only influenza A has been the sole devastating agent from the beginning and has proved its novelty through various rapid shifts and drifts, making it more virulent and adaptable for new species lacking any pre-existing immunity against it. Most of the recently isolated influenza viruses from pigs, however, have been H1N1 viruses. Sporadic human infections with swine flu have occurred initially in persons with direct exposure to pigs. ${ }^{8} 9$ The clinical spectrum of infection with the H1N1 virus is broad, and ranges from mild upper respiratory tract illness to severe complications such as pneumonia resulting in respiratory failure, Acute Respiratory Disease Syndrome (ARDS), multi-organ failure and death and mainly observed. In pigs, influenza infection produces fever, lethargy, sneezing, coughing, difficulty breathing and decreased appetite. In humans, symptoms include fever, cough, sore throat, body aches, headache, chills and fatigue. ${ }^{10}$ We can diagnose the swine flu by symptoms of acute febrile respiratory illness with laboratoryconfirmed by identifying antigens H1N1 influenza A virus through detection by real-time Reverse Transcriptase-Polymerase Chain Reaction (RT-PCR) or culture. In developing countries like Ethiopia it can causes great economic loss due to its treatment and control cost. However, we can control the impacts of swine flu using vaccination, use of antivirals for prophylaxis, use of antivirals for treatment and other simple measures like standard precautions, particularly respiratory and cough hygiene. ${ }^{11}$ Taking this fact into account, the objective of this study paper is:

a. To review the economic and public health importance of swine flu.

b. To overview the epidemiology of swine flu and its status in Ethiopia.

\section{Materials and methods}

This review work was carried out by undertaking a systematic review of the published research papers available online. Accordingly, sixty two published scientific research papers conducted on Swine flu reviewed and their extracted major findings were presented. The conclusion and recommendation of the reviewed papers and point out way forwards.

\section{Swine flu}

\section{Etiology}

Historical background of swine flu virus: Swine influenza was first proposed to be a disease related to human flu during the $1918 \mathrm{flu}$ pandemic, when pigs became sick at the same time as humans ${ }^{12}$ The first identification of an influenza virus as a cause of disease in pigs occurred about ten years later, in $1930 .{ }^{13}$ For the following 60years, 
swine influenza strains were almost exclusively H1N1. Then, between 1997 and 2002, new strains of three different subtypes and five different genotypes emerged as causes of influenza among pigs in North America. In 1997-1998, H3N2 strains emerged. These strains, which include genes derived by reassortment from human, swine and avian viruses, have become a major cause of swine influenza in North America. Reassortment between H1N1 and H3N2 produced H1N2. In 1999 in Canada, a strain of H4N6 crossed the species barrier from birds to pigs, but was contained on a single farm. ${ }^{14}$ The currently circulating strain of swine origin influenza virus of the H1N1 strain has undergone triple reassortment and contains genes from the avian, swine and human viruses. ${ }^{15}$ Swine influenza $A$ viruses are singlestranded RNA viruses with a segmented genome that belongs to the family of Orthomyxoviridae, genus Influenza virus A which has eight segments that encode eleven proteins. Influenza A viruses are classified into subtypes based on two surface glycoproteins, the hemagglutinin (HA) and neuraminidase (NA). ${ }^{16}$ The new influenza virus called H1N1 virus or (Hemagglutinin type 1) and N1 (neuraminidase type1) can mutate over time causing changes to the structure of surface proteins called antigens and infects humans. The virus that has a type 1 HA and type $2 \mathrm{NA}$, for example, would have the subtype H1N2. At least 16 types of hemagglutinins ( $\mathrm{H} 1$ to H16), and 9 neuraminidases (N1 to N9) are known to exist in birds, and two additional HA and NA types occur in bats while small subsets of avian subtypes circulate in other mammals including pigs. ${ }^{13,17-19}$ The HA, and to a lesser extent the NA, are major targets for the immune response, and there is ordinarily little or no cross-protection between different HA or NA types. The high variability is the result of two processes; mutation (antigenic drift) which causes gradual changes in the HA and NA proteins of the virus, and genetic reassortment (antigenic shifts) which can cause more rapid and abrupt changes. The new virus shares some characteristics of a pandemic strain, e.g. it can be transmitted from humans to humans, causes disease, people are not or only partially immune to the virus from previous infections and exposure results in productive infection. ${ }^{20}$

\section{Epidemiology}

Occurrence and geographical distribution: Swine influenza has been reported from North and South America (especially the United States, Midwest and Canada), Europe, parts of Asia and Africa. The viruses are thought to be enzootic in most areas that have dense populations of pigs, but they might remain undetected in some regions, as infected herds can be asymptomatic or have only mild clinical sig. ${ }^{21-23}$ Swine flu has been terror effects of all-round the globe and has been declared epidemic in most part of the world. The 2009-2010 human pandemic was caused by $a$ virus that appears to have resulted from genetic reassortment between North American and Eurasian swine influenza viruses. This virus now circulates in human populations worldwide. People have transmitted it to herds of pigs, and it has reassorted with various swine influenza viruses.

Transmission:Influenza A viruses can be directly transmitted from pigs to people and from people to pigs. Food is not yet known to be a vehicle for the transmission of this new influenza virus. The actual transmission is from pig to pig but swine flu virus can also jump from pig to human and once in human, it can spread from human to human very easily through respiratory secretions like coughing, sneezing, touching nose or mouth and contaminated surfaces via large-particle droplets. However, the swine influenza virus can be transmitted to humans via contact with infected pigs or environments contaminated with swine influenza viruses and then touching their nose or mouth. All bodily secretions are considered potentially infectious. ${ }^{27,28}$

\section{Pathogenesis}

The primary event after transmission of the virus is the invasion of the respiratory epithelium. The histopathological changes which were observed included epithelial cells damage, airway plugging and peribronchial and perivascular mononuclear cell infiltration. These sites of epithelial damage are often superinfected with bacteria, but severe lung damage can be caused by the virus. After the initial illness, the host usually mounts an immune response which involves a rise in antibody titers as well as T cell activation. The production of interferon in the respiratory mucosa is associated with a fall in virus shedding. Severe pneumonia with multifocal infiltrates and rapid progression to acute respiratory distress syndrome and multi-organ failure has been reported. Increased pathogenicity is accompanied by greater influx of neutrophils into lungs and increased protein expression of cytokines and chemokine in lung tissues. ${ }^{29,30}$

\section{Signs and symptoms}

Swine: Although the precise incubation period has not been established for pandemic H1N1 influenza $A$ infection, it could range from one to seven days, and most likely from one to four days. Illness may be seen only in certain age groups, while other animals remain asymptomatic. The clinical signs may include fever, lethargy, anorexia, weight loss, coughing, sneezing, nasal and ocular discharge, conjunctivitis, listless behavior and labored breathing (expiratory dyspnea or "thumping") $18,31-34$

Human:The United States Centre's for Disease Control and Prevention includes symptoms of swine flu infection are fever, cough, sore throat, diarrhea, vomiting, myalgia and joint pains. Patients with 2009 influenza A H1N1 infections have higher rates of gastrointestinal symptoms and less fever compared with those who have seasonal flu. The most common causes of death due to swine-flu are respiratory failure, pneumonia, sepsis, dehydration (from excessive vomiting), high fever and electrolyte imbalance. ${ }^{35}$

\section{Diagnosis}

Cases of swine flu are divided into confirmed, probable or suspected cases. Influenza-like Illness (ILI) is defined as fever and a cough and/or a sore throat in the absence of a known cause other than influenza. For diagnosis of swine influenza $A$ infection, respiratory specimen (nasopharyngeal swab, throat swab nasal aspirate, nasal washing) would generally need to be collected within the first 4 to 5 days of illness (when an infected person is most likely to be shedding virus) for laboratory diagnosis. ${ }^{9}$

Reverse transcriptase polymerase chain reaction: Based on the publically released hemagglutinin sequences of the currently circulating virus, real-time RT-PCR assays have been developed. The RNA extracted from nasopharyngeal aspirates, throat swabs and bronchial aspirates is amplified and detected by this assay. This assay was seen to be highly specific for the swine-origin $\mathrm{H} 1 \mathrm{~N} 1$ virus and was able to distinguish this from the seasonal H1N1 as well as on H1N1 organisms and are rapid. ${ }^{36}$

Rapid diagnostic tests:The use of rapid diagnostic tests to detect antigens of the virus was compared with the standard RT-PCR in 65 patients and it was observed that the method had $60 \%$ to $80 \%$ sensitivity. These findings indicate that although a positive test suggests diagnosis of H1N1 influenza, a negative result does not rule out the same. Besides this, the test often requires a high virus concentration in the respiratory secretions, and if negative, its results are interpreted based on the clinical suspicion of illness. The comparison of antibody 
titer during the acute illness and 10 to 14 days later will help make a diagnosis too but the test is not used for diagnostic purposes. ${ }^{37}$

Viral culture: The virus when grown on culture helps one make a $100 \%$ specific diagnosis. But it is not rapid and it takes a time. The sensitivity and negative predictive value is also quite high at around $90 \%$. The virus grows on chick embryo as well as monkey kidney cell cultures within 48 to 72 hours of its inoculation. ${ }^{38}$

Other tests:Hematological and biochemical testing may suggest leucopenia, elevated lactate dehydrogenase and creatine kinase. Uncommonly, thrombocytopenia may also occur and chest $\mathrm{X}$-ray abnormalities may be noted particularly in those severely affected and hospitalized. Neutrophil to Lymphocyte ratio less than or equal to 2 appears to be a marker to identify those likely to have H1N1 infection and used as a screening tool. ${ }^{39,40}$

\section{Zoonotic importance of swine flu}

Even though eating of swine meat does not transmit the disease, the transmission of swine influenza from swine to human is believed to occur mainly in swine farms where farmers are in close contact with live pigs. In 2009, a novel H1N1 virus emerged in human populations. It causes respiratory and gastrointestinal symptoms in human with death related to other bacterial complications. ${ }^{41-43}$ Infections with various $\mathrm{H} 1 \mathrm{~N} 1, \mathrm{H} 3 \mathrm{~N} 2$ and $\mathrm{H} 1 \mathrm{~N} 2$ swine influenza viruses are reported sporadically in humans. The more recent study also suggested seropositivity to H1N2 SIV among swine farmers and veterinarians. Healthcare workers have acquired H1N1 in occupational settings. ${ }^{44,45}$

\section{Economic importance of swine flu}

During the current 2009 H1N1 pandemic, though the studies clearly confirmed that eating pork and other pig meat do not cause the disease, the terror among the people led to huge economic losses to the pig industry worldwide. Other areas that might be affected during a pandemic are International tourism, industries, healthcare systems, educational institutions, and entertainment industry. The cost of prevention and control of a pandemic along with the compensations paid by the governments through the world during a pandemic is also very high. Abortion and reduced growth rates in young pig causes a remarkable economic loss. As on 19 October 2009, more than 414 000 cases and about 5000 deaths had been reported to WHO by 195 countries. The global economic impact has been estimated to be US $\$ 800$ billion with anticipated quarantines and interruption in global trade suspension of international trade and travel. ${ }^{46-48}$

\section{Treatments}

In swine:Antibiotics may be used to control secondary infections. Antiviral drugs used in human influenza treatment are not generally administered to swine. As swine influenza is rarely fatal to pigs, little treatment beyond rest and supportive care is required. ${ }^{49}$

In Human:Two classes of antiviral drugs are available for the prevention and treatment of influenza: neuraminidase inhibitors and adamantine, which inhibit a viral protein. Currently circulating 2009 H1N1 virus is susceptible to Oseltamivir (Tamiflu) and Zanamivir (Relenza) but resistant to adamantine (Amantadine and Rimantadine). These drugs reduce the median duration of symptoms by approximately one day and reduce the chance of contracting influenza by 70 to $90 \%$ when used for known influenza exposure. Zanamivir and Oseltamivir are structurally related drugs that have been approved by the United States Food and Drug Administration (USFDA) for the prophylaxis and treatment of influenza. In addition to their activity against current influenza A and influenza B strains, they are also active against the strain that caused the 1918 pandemic and against avian influenza a strains. ${ }^{50}$ Ayurveda promotes the concept that if one's immune system is strong, then even if the body is exposed to viruses, one will not be affected. During a pandemic or an epidemic, it emphasizes on the immunity of people living in regions affected by viruses. This branch of medicine promotes the intake of special herbs to increase the immunity level of the people. Ayurveda remedies comprise pure natural herbs like basil, garlic, gooseberry, tulsi, aloe vera and ginger which are effective in preventing swine flu. Moreover, the herbs are used to relieve swine flu symptoms, and boost the immune system against the $\mathrm{H} 1 \mathrm{~N} 1$ virus $^{.51}$

\section{Prevention and control}

Prevention and control of swine influenza has three components namely; prevention in swine, prevention of transmission to humans, and prevention of its spread among humans. Novel Swine flu can be prevented with or without vaccines. In swine the spread of influenza can be prevented by facility management and herd management. Sanitation helps to prevent transmission on fomites and mechanical vectors. All-in/all-out program also helps to prevent the introduction of viruses. ${ }^{52}$ From a public health standpoint, for a vast majority of populations in developing and resource-constrained countries, pharmacological interventions such as vaccines and antivirals are not likely to play a major role at present, due in part to limited supply, lack of access and the high costs involved. Such countries should have to depend on various non-pharmaceutical interventions. Personal hygiene, including hand hygiene, if observed properly, can be effective in preventing respiratory viral infections, as shown by empirical studies. Alcohol-based gel or foam hand sanitizers work well to destroy viruses and bacteria. Surfaces, which can be done effectively diluted with chlorine, bleach solution. ${ }^{53-56}$

\section{Swine flu vaccines}

It has been difficult to develop a vaccine for H1N1 influenza $A$ virus that provides long lasting immunity. This is due to the antigenic drift of the virus where the circulating strain in an infectious cycle is different from the previously circulating strain and influenza vaccine antigens end to be updated frequently. The objective of vaccinating swine is to reduce the clinical impact of the influenza virus in the pigs, to reduce contagion within the pig facility, and to reduce the risk of worker infection. A vaccine against H1N1 influenza is available, although supplies were limited initially. Currently, there are two types of vaccine available (pandemic and calve pain) in 5 compositions: the nasal vaccine, $0.25 \mathrm{ml}$ shot, $0.5 \mathrm{ml}$ shot and prefilled $0.5 \mathrm{ml}$ syringe without additive and multilevel latex-free shot. The nasal vaccine has a weakened ("attenuated") virus and is administered by sniffing. Although the current seasonal influenza vaccine contains a component effective against another A (H1N1) virus, it is not effective against the 2009 pandemic influenza $A$ (H1N1) virus. ${ }^{57}$

\section{Status of swine flu in ethiopia}

According to global status of influenza in 2010, 12 cases of swine influenza in Ethiopia were reported. ${ }^{58}$ Ministry of Health $(\mathrm{MOH})$, Ethiopian Health and Nutrition Research Institute (EHNRI) and CDC-Ethiopia jointly conducted a national workshop on influenza surveillance systems, functions and its operational components in such a way that able to implement influenza surveillance in different regions. ${ }^{59}$ In March 2016, a total of 13 patients complaining of Influenza-like Illness (ILI) or Severe Acute Respiratory Infections (SARI) were reported with throat swab samples from 
predesigned influenza sentinel sites. Among them four were tested positive for Influenza a H1N1 2009 pandemic and three were positive for seasonal influenza A (H3N2). Starting from October 2015, the positivity rate of Pandemic Influenza H1N1 is increasing. In Ethiopia, currently there are confirmed cases with no death associated with the condition. The virus has been detected on February 4, in different hospitals. However, sources in the Ministry of Health say the subtype of the influenza detected in Addis Ababa is less dangerous than others and Ethiopia has testing and treating capabilities. Test samples are sent to the Center for Disease Control (CDC) of United States for further investigation as a matter precaution. ${ }^{60}$

\section{Conclusion and recommendations}

Swine influenza is a respiratory disease caused by influenza viruses that infect the respiratory tract of pigs and result in nasal secretions, a barking-like cough, decreased appetite, and listless behavior in pigs and causes sudden cough and fever in human which can be identified clinically and/or by laboratory tests. It has become the world's fastest moving influenza, as it is pandemic, sweeping across many countries in a short period of time because of aerosol transmission between persons and swine. It causes a high economic loss and public health significance. It can be prevented by antiviral therapy and vaccines. It can be a great fear for developing countries such as Ethiopia because of its contagiousness and high cost of its treatment and control, when observed. Therefore, the following recommendations will be forwarded;

a. Overcrowding of pigs and close contact with them should be reduced.

b. Victims like swine farm owners and veterinarians should be vaccinated.

c. Awareness about the disease should be given for the societies.

d. Persons from outside the country should always be checked as free of swine flu at airport.

e. If observed it should be reported immediately to the authorities.

\section{Acknowledgments}

None.

\section{Conflicts of interest}

Author declares there are no conflicts of interest.

\section{Funding}

None.

\section{References}

1. Silva CS, Mullis LB, Pereira O, et al. Human Respiratory Coronaviruses Detected In Patients with Influenza Like Illness in Arkansas, USA. Virol Mycol. 2014;2014(Suppl 2):004.

2. Awaidy ST, Obeidani IA, Singh JV, et al. Epidemiology of Pandemic H1N1 in Oman and Public Health Response 2009. J Community Med Health Educ. 2015;5:343.

3. G Aringnestad, K Vainio, O Hungnes. Does Virus Interference Accoun for the Multiple Epidemic Waves that are Characteristic of the 1918 Spanish Influenza and other Influenza Pandemics? J Anc Dis Prev Rem. 2015;3:121.

4. Heidi A Davidson, Erin Kennedy, Michael A Jhung, et al. Knowledge, Attitudes and Practices Regarding 2009 H1N1 Vaccine Among Patients Hospitalized With Influenza A(H1N1) pdm09. Virol Mycol. 2014;S2:002
5. Sips GL, Pekosz A, Huckriede A, et al. Interaction of Influenza A/ H1N1pdm Virus with Human Neuronal and Ocular Cells. Virol Mycol. 2014;S2:001.

6. Dawood FS, Jain S, Fineli L, et al. Novel Swine-Origin Influenza A (H1N1) Virus Investigation Team. Emergence of a novel swine-origin Influenza A(H1N1) virus in humans. NEngl J Med. 2009;360:26052615.

7. Myers KP, Olsen CW, Setterquist SF, et al. Are swine workers in the United States at increased risk of infection with zoonotic influenza virus. Clin Infect Dis. 2006;42(1):14-20.

8. Hause BM, Ducatez M, Collin EA, et al. Isolation of a novel swine influenza virus from Oklahoma in 2011 which is distantly related to human influenza C viruses. PLoS Pathog. 2013;9(2):e1003176.

9. Mahesh SD, Shantavan SS, Borse LB, et al. A Review on Swine Flu. Pharma Science Monitor . 2015;6(1):308-324.

10. Kothalawala H, Toussaint MJ, Gruys E. An Overview of Swine Influenza. VetQ. 2006;28(2):46-53.

11. Nuno M, Chowell G, Gumel AB. Assessing the role of basic control measures, antivirals and vaccine in curtailing pandemic influenza: scenarios for the US, UK and the Netherlands. $J R$ Soc Interface. 2007;4(14):50-521.

12. Knobler S, Mack A, Mahmoud A, et al. The Threat of Pandemic Influenza: Are We Ready? Workshop Summary, National Academies Press, USA. 2005

13. Olsen CW. The Emergence of Novel Swine Influenza Viruses in North America. Virus Research. 2002;85(2):199-210.

14. Taubenberger JK, Morens DM. Influenza: the mother of all pandemics. Emerg Infect Dis. 2006;12(1):15-22.

15. Newman AP, Reisdorf E, Beinemann J, et al. Swine influenza A (H1N1) triple reassortant virus infection, Wisconsin. Emerging Infectious Diseases. 2008;14(9):1470-1472.

16. Brockwell Staats C, Webster RG, Webby RJ. Diversity of Influenza Viruses in Swine and the Emergence of a Novel Pandemic Influenza A (H1N1), Influenza. Other Respi Viruses. 2009;3(5):207-213.

17. OIE. Manual of diagnostic tests and vaccines for terrestrial animals. (6th edn), OIE, Paris. 2008

18. OIE. Manual of diagnostic tests and vaccines for terrestrial animals Paris, OIE; Swine influenza. 2010.

19. Tong S, Zhu X, Li Y, et al. New world bats harbor diverse influenza A viruses. PLoS Pathog. 2013;9(10):e1003657.

20. Ma W, Richt JA. Swine influenza vaccines, current status and future perspectives. Anim Health Res Rev. 2010;11(1):81-96.

21. Vincent AL, Ma W, Lager KM, et al. Swine influenza viruses a North American perspective. $A d v$ Virus Re. 2008;72:127-154.

22. Zhu H, Webby R, Lam TT, et al. History of swine influenza viruses in Asia. Curr Top Microbiol Immunol. 2013; 370:57-68

23. Brown IH. History and epidemiology of swine influenza in Europe. Curr Top Microbiol Immunol. 2013;370:133-146.

24. Tremblay D, Allard V, Doyon JF, et al. Emergence of a new swine H3N2 and pandemic (H1N1) 2009 influenza A virus reassortant in two Canadian animal populations, mink and swine. $J$ ClinMicrobiol. 2011;49(12):4386-4390.

25. Choi YK, Pascua PN, Song MS. Swine influenza viruses: an Asian perspective. Curr Top MicrobiolImmunol . 2013;370:147-72.

26. Krumbholz A, Lange J, Durrwald R, et al. Prevalence of antibodies to European porcine influenza viruses in humans living in high pig density areas of Germany. Med Microbiol Immunol . 2014;203(1):13-24. 
27. Maines TR, Jayaraman A, Belser JA, et al. Transmission and pathogenesis of swine-origin $2009 \mathrm{~A}(\mathrm{H} 1 \mathrm{~N} 1)$ influenza viruses in ferrets and mice. Science. 2009;325(5939):484-487.

28. Steel J, Lowen AC, Pena L, et al. Live attenuated influenza viruses containing NS1 truncations as vaccine candidates against H5N1 highly pathogenic avian influenza, $J$ Virol. 2009;83(4):1742-53.

29. Swayne DE. Avian influenza. Foreign animal diseases. United States Animal Health Association, USA. 2008. p.137-146

30. Dolin R. Influenza. In: Kasper DL (Eds.), Harrisson's principles of internal medicine, McGraw Hill, USA. 2005. p.1066-1070.

31. Acha PN, Szyfres B. Zoonoses and communicable diseases common to man and animals Chlamydiosis, rickettsioses and viruses. (3rd edn), Pan American Health Organization, USA. 2003;3:1-66.

32. Van RK. Avian and swine influenza viruses: our current understanding of the zoonotic risk. Vet Res. 2007;38(2):243-260.

33. Lokwani P, Kumar P, Upadhyay Y, et al. Swine Flu: An Overview. Journal of Applied PharmaceuticalScience. 2011;1(4):29-34.

34. Janke BH. Clinicopathological features of swine influenza. Curr Top Microbiol Immunol. 2013;370:69-83.

35. Parmar S, Shah N, Kasarwala M,et al. A Review on Swine Flu. JPSBR 2011;1(1):11-17.

36. Poon LLM, Chan KH, Smith GJ, et al. Molecular detection of a novel human influenza $(\mathrm{H} 1 \mathrm{~N} 1)$ of pandemic potential by conventional and RealTime Quantitative RT-PCR assays. Clin Chem. 2009;55(8):1555-1558.

37. Beigel J, Bray M. Current and future Antiviral Therapy of severe seasonal and avian influenza. Antiviral Res . 2008;78(1):91-102.

38. Ginocchio CC, Zhang F, Manji R, et al. Evaluation of multiple test methods for the detection of the novel 2009 influenza A (H1N1) during the New York City outbreak. J Clin Virol. 2009;45(3):191-195.

39. Perez-Padilla R, de la Rosa-Zamboni D, Ponce de Leon S, et al. Pneumonia and respiratory failure from swine-origin influenza A (H1N1) in Mexico. N Engl J Med. 2009;361(7):680-689.

40. Vijaydeep Siddharth, VineetGoyal, Vipin Kumar Koushal. Clinical Epidemiological Profile of Influenza A H1N1 Cases at a Tertiary Care Institute of India. Indian J Community Med . 2012;37(4):232-235.

41. Garten RJ, Davis CT, Russell CA, et al. Antigenic and genetic characteristics of swine-origin 2009 A(H1N1) influenza viruses circulating in humans. Science. 2009;325(5937):197-120.

42. Trifonov V, Khiabanian H, Greenbaum B, et al. The origin of the recent swine influenza A (H1N1) virus infecting humans. Euro Surveill. 2009;14(17).

43. Christman MC, Kedwaii A, Xu J,et al. Pandemic (H1N1) 2009 virus revisited: an evolutionary retrospective. Infect Genet Evol. 2011;11(5):803-811.
44. Myers PK, Olsen CW, Gray GC. Cases of swine influenza in humans: a review of the literature. Clin Infect Dis. 2007;44(8):1084-1088.

45. Epperson S, Jhung M, Richards S, et al. Human infections with influenza A (H3N2) variant virus in the United States. Clin Infect Dis. 2013;57(1):S4-S11.

46. Sander B, Nizam A, Garrison LP, et al. Economic Evaluation of Influenza Pandemic Mitigation Strategies in the United States Using a Stochastic Microsimulation Transmission Model. Value Health. 2008;12(2):226-233.

47. Vincent AL, Ma W, Lager KM, et al. Characterization of a newly emerged genetic cluster of $\mathrm{H} 1 \mathrm{~N} 1$ and $\mathrm{H} 1 \mathrm{~N} 2$ swine influenza virus in the United States. Virus Genes. 2009;39(2):176-185.

48. Schultz Cherry S, Olsen CW, Easterday BC. History of swine influenza. Curr Top Microbiol Immunol. 2013;370:21-28.

49. Dee SA, Aiello SE, Moses MA. Swine influenza. The Merck veterinary manual Whitehouse Station, USA. 2012.

50. Tumpey TM, Garcia-Sastre A, Mikulasova A. Existing Antiviral Are Effective Against Influenza Viruses with Genes from the 1918 Pandemic Virus. ProcNatl Acad Sci. 2002;99(21):138-149.

51. Priya J. Swine flu-Ayurveda. Buzzle, USA. 2009

52. CDC. Novel influenza A (H1N1) virus infections among health-care personnel, United States. Morb Mortal Wkly Rep. 2009;58(23):641-645.

53. Ansari SA, Springthorpe VS, Sattar SA, et al. Potential role of hands in the spread of respiratory viral infections: Studies with human parainfluenza virus 3 and rhinovirus 14. J Clin Microbiol. 1991;29(10):2115-2119.

54. Cairncross S. Handwashing with soap a new way to prevent Acute Respiratory Infections. Trop Med Int Health. 2003;8:677-679.

55. Ramirez A, Capuano AW, Wellman DA, et al. Preventing zoonotic influenza virus infection. Emerging Infect. Dis. 2006;12(6):996-1000.

56. Dandagi LG, Byahatti SM. An insight into the swine-influenza A (H1N1) virus infection in humans, Lung India. 2011;28(1):34-38.

57. Pandemic (H1N1) WHO, Switzerland. 2009

58. Gideon. Influenza Global Status report. Gideon Informatics, USA. 2016. p.5-40.

59. An Independent review of Pharmaceutical News and Issues. EPA, Addis Ababa, Ethiopia. 2009; 15(1):12.

60. Abyot B. Weekly Epidemiological Bulletin. Swaziland Street, Addis Ababa, Ethiopia. 2016;2(10):1-9. 